\title{
Local WMR Navigation with Monocular Data
}

\author{
Lluis Pacheco, Ningsu Luo, Xavier Cufi and Javier Cobos \\ Depts of Computer Engineering and Electronics and Control Engineering \\ University of Girona \\ Girona, Spain \\ \{lluispa,ningsu,xcuf,jcobosg\}@eia.udg.edu
}

\begin{abstract}
This article presents recent WMR (wheeled mobile robot) navigation experiences using local perception knowledge provided by monocular and odometer systems. A local narrow perception horizon is used to plan safety trajectories towards the objective. Therefore, monocular data are proposed as a way to obtain real time local information by building two dimensional occupancy grids through a time integration of the frames. The path planning is accomplished by using attraction potential fields, while the trajectory tracking is performed by using model predictive control techniques. The results are faced to indoor situations by using the lab available platform consisting in a differential driven mobile robot.
\end{abstract}

Keywords-Mobile robot navigation, local path planning, model predictive control, monocular perception techniques

\section{INTRODUCTION}

Autonomous robot navigation without GPS is an interesting research topic faced by navigators [1]. Therefore, indoor WMR (wheeled mobile robot) navigation using computer vision techniques are normally based on environment description. The data analysis can be considered as a map when it is provided enough information to reach robot localizations. Normally, navigation based on maps includes geometric environmental information. The first research was developed using $2 \mathrm{D}$ environment models consisting of a free or occupied grid [2]. The virtual potential field idea improved performance by using objective attraction and obstacle repulsion forces [3]. Therefore, WMR navigation strategies should be planned so that obstacle collisions are avoided during navigation towards the objective. The meaningful navigation idea consists of providing feasible and expected visual landmarks, thereby allowing the robot's map position to be calculated using information about recognized landmarks. Localization of the WMR using machine vision system computation is done by using sensor information from where landmark detection is correlated with the corresponding map position. Then, the robot position and orientation are estimated using the previous data. WMR localization can be considered as absolute or incremental [4]. Incremental navigation methods consider the initial robot coordinates as known; machine vision system information is used as a way to improve the robot's positioning. Otherwise, absolute navigation methods would not know the initial robot coordinates. When absolute localization is used, the navigation system should build a correspondence between the WMR's perception information and the database. Estimations based on sensor uncertainty probability allow better robot localization. The ambiguities can be solved by using statistical methods [5]. The incremental localization can use three different techniques: localization based on geometrical representation, localization based on topological space representation, or localization based on landmark detection [6],[7] and [8]. The different presented methods differ in the map representation however all share the need of landmark search and detection that allow computing the robot localization. Furthermore, other research work studying natural agents has presented a new robot navigation paradigm. Hence, corridor planning for natural agents has been presented as a new and useful robot control and planning framework using low level obstacle avoidance and simple control [9]. Butler has developed a system in which innate local navigation animal abilities are combined with a very simple imposed path-like structure to produce a desired overall motion. The developed research showed that local animal abilities such as control and perception can be combined with a very simple imposed path-like structure to produce the desired overall motion. Hence, path-planning in partially unknown environments should be short enough to allow local reactive behaviours; consequently long trajectory planning should be flexible in order to deal with short term uncertainties while the global task is accomplished. This article explores this challenge as a navigation strategy for the available WMR platform PRIM that consists in a differential driven one with a free rotating wheel [10]. Hence, in this work, local animal abilities are performed by using a simple perception system, which consists of a monocular camera and an odometer system. Moreover, the field of view is constrained to the WMR neighbourhood; thus only few seconds of trajectory planning can be done. The simple corridor structure is faced up by using local attraction potential fields; where MPC (model predictive control) techniques are used to perform accurate trajectory tracking. Therefore, an example in indoor scenarios and the path followed is reported. 


\section{LOCAL PERCEPTION HORIZON}

\section{A. Introduction}

Nowadays camera systems have a set of advantages when are compared with other range systems. Hence, they allow a richer scene description while the prices are decreasing and computing power is increased. The computer vision techniques applied to WMR have solved the problem of obstacle detection by using different methods as stereo vision, optical flow or DFF (depth from focus). Stereo vision systems seem to provide the easiest cues to infer scene depth [11]. The optical flow techniques used in WMR result in several applications as i.e. structure knowledge, obstacle avoidance, or visual servoing [12]. The DFF methods are also suitable for WMR. For example, three different focused images were used, with almost the same scene, acquired with three different cameras [13]. In this work, it is supposed that available obstacle positions are provided by using computer vision systems.

In this context, the allowed navigation control signals should achieve the obstacle avoidance politics as well as the final desired coordinates. Scientific community has developed several studies in this field. Based on the dynamic window approach with available robot speeds, the reactive avoidance collisions, safety stop and goal can be achieved using the dynamic constraints of WMR [14]. Some approaches on mobile robots propose the use of potential fields, which satisfy the stability in a Lyapunov sense, in a short prediction horizon [15]. This paper proposes the use of the visual information as a dynamic window where the collision avoidance and safety stop should be planned. Thus, local visual data are used to plan safety trajectories that approach the WMR to the desired configuration that acts as an attraction potential field.

The use of sensor information as a useful source to build 2D environment models consists of a free or occupied grid was proposed in [2]. The occupancy grid knowledge has been used for static indoor mapping with a 2D grid [16]. In this work it is proposed the use of the local narrow visual information available from the camera as a local map that has enough information to achieve an objective configuration. The occupancy grid can be obtained in real time by using computer vision methods and odometer data. Hence, once the local information is obtained, it is proposed an algorithm that computes the visual local desired coordinates as well as the trajectory to be reached. The research developed assumes indoor environments as well as flat floor constraints. However, it can be also applied in outdoor environments. This section firstly presents the local map relationships with the camera configuration and poses. Then, the navigation strategy is presented, which uses the monocular perception data as a horizon of perception where the objective configuration acts as an attraction potential field. Finally, some physical and dynamical constraints are analyzed as well as local minimal failures.

\section{B. The scene perception}

The local visual data provided by the camera are used to plan a feasible trajectory and to avoid the obstacle collision. The scene available coordinates appear as an image, where each pixel coordinates correspond to a 3D scene coordinates. Hence, scene coordinates can be computed using camera setup and pose knowledge, and assuming projective perspective. The Figure 1 shows the robot configuration studied in this work.

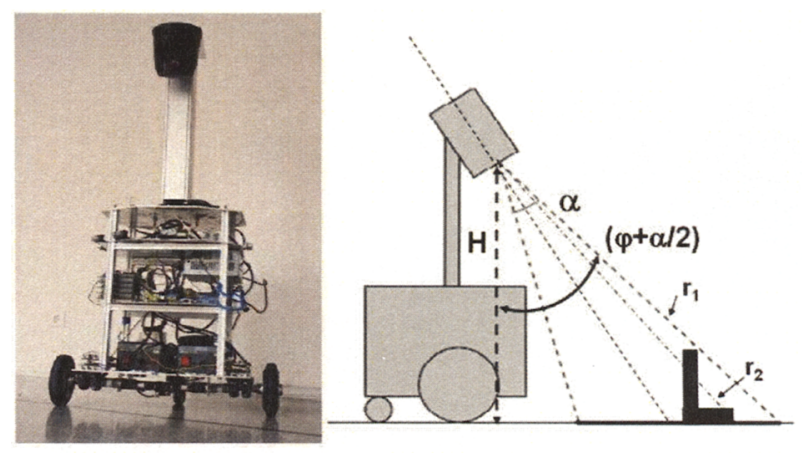

Fig. 1 Robot Configuration

The angles $\alpha, \beta$ and $\varphi$ are related to the vertical and horizontal field of view, and the tilt camera pose, respectively. The vertical coordinate of the camera is represented by $H$.

$$
\begin{array}{lll}
y_{j}=H \tan (\varphi-\alpha / 2+\Delta \alpha) & \Delta \alpha=\mathrm{K}_{\mathrm{j}} \frac{\alpha}{\mathrm{R}} & \left(0 \leq \mathrm{K}_{\mathrm{j}} \leq R\right) \\
x_{i, j}= \pm \frac{H}{\cos (\varphi-\alpha / 2+\Delta \alpha)} \tan (\Delta \beta) & \Delta \beta=\mathrm{K}_{\mathrm{i}} \frac{\beta}{\mathrm{C}} & \left(0 \leq \mathrm{K}_{\mathrm{i}} \leq C / 2\right)
\end{array}
$$

The $K_{i}$ and $K_{j}$ are parameters used to cover the image pixel discrete space. Thus, $R$ and $C$ represent the image resolution through the total number of rows and columns. It should be noted that for each row position, which corresponds to scene coordinates $y_{j}$, there exist $C$ column coordinates $x_{i, j}$. The above equations provide the available floor coordinates when no obstacle is detected, see Figure 2.

The available information provided by the camera is considered as a local horizon where the trajectory is planned. Hence, a local map with free obstacle coordinates is provided. It is noted that low resolution scene grids are used in order to speed up the computing process. It is also suggested to speed up the computing process based on a previously calculated LUT, (look up table), with the scene floor coordinates corresponding to each pixel.

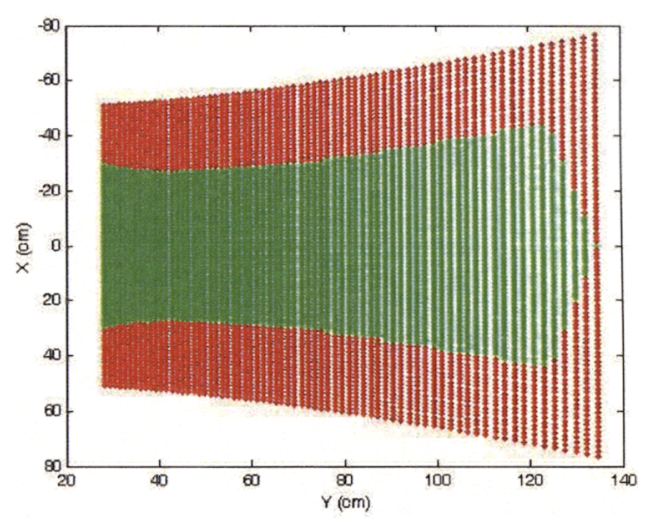

Fig. 2. Free of obstacles local on-robot map. The green cells depict the available floor coordinates. The red coordinates show the non available cells due to the mobile robot physical dimensions. 


\section{Attraction potential fields and local cell selection}

The local visual data provided by the camera are used to plan a feasible trajectory. The occupancy grid is obtained as a binary image, in which the obstacle blob areas and maximal size coordinates are computed. Using these data, the local visual desired coordinates are computed by considering the maximum geometric size of the closer obstacle and the objective coordinates that act as an attraction potential field. In this context the WP (wide-path) and the closer obstacle significant vertex are considered. When the selected position, due to the physical size of WMR, falls outside the available field of view, a turning action towards the direction of the desired coordinates is performed by commanding an available cell within the closer $Y$ column. Otherwise, when no obstacle is found, the procedure consists in selecting the optimal approaching cell.

It is relevant to be aware of the narrow field of view considered in this research. In this sense, a new advancing trajectory will produce a new environment description. In the human case it can be understood as an "enlighten my steps strategy" (as i.e. by using a small torch when we are exploring an unfamiliar dark place). It should be pointed the importance of selecting effective potential fields. The problem is formulated as finding the optimal cell that brings the WMR close to the desired coordinates $\left(x_{d}, y_{d}\right)$ by searching for the closest local desired coordinates $\left(x_{l d}, y_{l d}\right)$ within the available local coordinates $\left(x_{i j}, y_{j}\right)$. In this sense, perception is considered as a local receding horizon in which the trajectory is planned. The local desired cell is obtained by minimizing a cost function $J$ that should act as a potential field corridor. Thus, the cost function is minimized by attracting the robot to the desired objective through the free available local cell coordinates. Due to the narrow field of perception, the robot should orient towards the goal. Hence, the $\theta_{l g}$ value depicts the maximal angle that can be attained within the available local grid. Thus, first, the orientation error towards the goal should be minimized:

$$
\begin{aligned}
& \text { If } a \tan \left(y_{d} / x_{d}\right)=\theta_{d}>\theta_{l g} \\
& J_{1}(i, j)=\min \left(a b s\left(a \tan \left(y_{1} / x_{i 1}\right)-a \tan \left(y_{d i} / x_{d}\right)\right)\right) \quad \forall 1 \leq i \leq R, j=1
\end{aligned}
$$

Once the WMR is oriented towards the goal, when no obstacles are met, the Euclidean distance is proposed as approaching potential field:

$$
\begin{aligned}
& \text { If } a \tan \left(y_{d} / x_{d}\right)=\theta_{d} \leq \theta_{\mathrm{lg}} \\
& J_{2}(i, j)=\min \left(\left(x_{i j}-x_{d}\right)^{2}+\left(y_{j}-y_{d}\right)^{2}\right)^{1 / 2} \\
& \forall 1 \leq i \leq R, 1 \leq j \leq C
\end{aligned}
$$

When obstacles are met, the left and right closer obstacle vertex coordinates, increased by the robot WP (wide-path), are tested within expression (4). Thus, the vertex that produces minimal cost function is selected. The desired local coordinates are considered as final points, until not any new optimal local desired coordinates are provided.

\section{Local minimal failures and constraits}

The local minimal failures will be produced when a convergence criterion is not satisfied. Thus, the local visual information cannot provide with closer optimal desired coordinates, because obstacles are blocking the trajectory to the goal. In these situations, obstacle contour tracking may be proposed. Hence, local objectives for contour tracking are used, instead of the goal coordinates, as the source for obtaining a path until the feasible goal trajectories are found. The Figure 3 shows an example with local minimal failures. It is seen that at $\mathrm{A}$, the optimal trajectory is a straight line between $\mathrm{A}$ and $\mathrm{E}$. However, an obstacle is met at $\mathrm{B}$, and local minimal failure is produced at $\mathrm{B}$. When this is produced, no trajectory can approach to the desired goal, $\left(x_{d}, y_{d}\right)$. Then, obstacle contour tracking is proposed between B and C. Once C is attained, local minimization along coordinates $y_{j}$ is found and the trajectory between $\mathrm{C}$ and $\mathrm{D}$ is planned. From $\mathrm{D}$ to $\mathrm{E}$ local minimums are reached until the final goal is achieved. It should be noted that once $\mathrm{B}$ is reached, the left or right obstacle contour are possible. However, the right direction will bring the robot to an increasing $y_{j}$ distance. The robot follows the desired goals except when the situation of obstacle contour tracking is produced, and then local objectives are just the contour following points that can be commanded as attraction potential fields. Hence, it is proposed the use of effective attraction fields as well as feasible maps and landmarks to provide loca objective coordinates that can be used for guiding the WMR to reach the final goal coordinates.

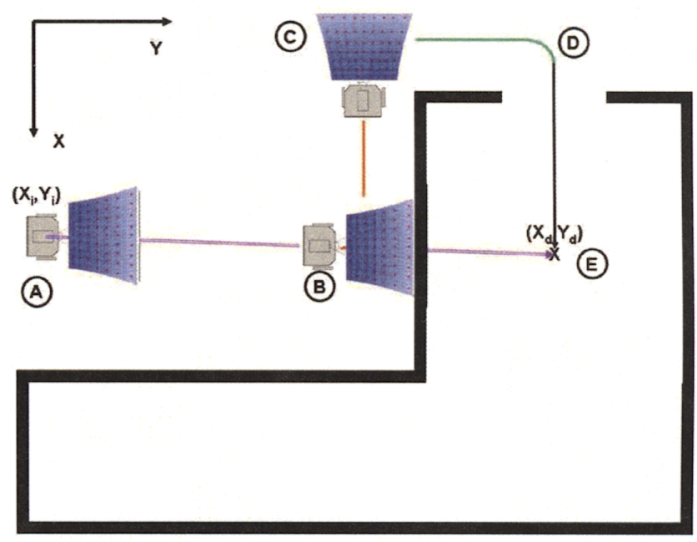

Fig. 3. Example with local minimal failures

The physical robot size reduces the horizon of available coordinates by the path width of WMR, as it is shown in Figure 2. Some important WMR dynamic aspects, as reactive and stop distances, are also considered. The dynamic reactive distance should be enough to plan safety trajectories; it is related to the robot dynamics and the processing time for each frame. Thus, by using the three models corresponding to the WMR PRIM, three different dynamic reactive distances are found. The Table 1 shows these concepts, when it is supposed mobile obstacles until $0.5 \mathrm{~m} / \mathrm{s}$ and a perception speed of 4 frames each second. 
Table 1. Reactive criterion and minimal allowable distances

\begin{tabular}{ccccc}
\hline Model & $\begin{array}{c}\text { Safety } \\
\text { stop } \\
\text { dist. }\end{array}$ & $\begin{array}{c}\text { Obstacle } \\
\text { reactive } \\
\text { distances }\end{array}$ & $\begin{array}{l}\text { Robot } \\
\text { displacement }\end{array}$ & $\begin{array}{c}\text { Minimal } \\
\text { allowable } \\
\text { distances }\end{array}$ \\
\hline $\begin{array}{c}\text { Low velocities } \\
(0.15 \mathrm{~m} / \mathrm{s})\end{array}$ & $0.085 \mathrm{~m}$ & $0.125 \mathrm{~m}$ & $0.038 \mathrm{~m}$ & $0.248 \mathrm{~m}$ \\
\hline $\begin{array}{c}\text { Medium } \\
\text { Velocities } \\
(0.3 \mathrm{~m} / \mathrm{s})\end{array}$ & $0.178 \mathrm{~m}$ & $0.125 \mathrm{~m}$ & $0.075 \mathrm{~m}$ & $0.378 \mathrm{~m}$ \\
\hline $\begin{array}{c}\text { High Velocities } \\
(0.45 \mathrm{~m} / \mathrm{s})\end{array}$ & $0.310 \mathrm{~m}$ & $0.125 \mathrm{~m}$ & $0.113 \mathrm{~m}$ & $0.548 \mathrm{~m}$ \\
\hline
\end{tabular}

Hence, the allowed visual trajectory distance will set the speed that can be reached. The next section introduces the MPC techniques used that guarantee a convergence to the visual local desired coordinates until is not commanded the next goal position.

\section{TRAJECTORY TRACKING AND LOCAL MODEL PREDICTIVE CONTROL}

\section{A. Introduction}

The minimization of path tracking error is considered as a challenging subject in mobile robotics. The main objective of highly precise motion tracking consists in minimizing the error between the robot and the desired path. Real-time implementation of MPC (model predictive control) in the mobile robotics has been developed using global vision sensing [17]. In [18], MPC based optimal control was studied, which is useful for cases when nonlinear mobile robots are used under several constraints. In general, real-time implementation is possible when a short prediction horizon is used. By using MPC, the idea of receding horizon can deal with the local sensor information. MPC is based on minimizing a cost function related to the objectives for generating the optimal inputs. The LMPC (local model predictive control) is proposed to use the available visual data in the navigation strategies for the goal achievement [19]. It is defined the cost function as follows:

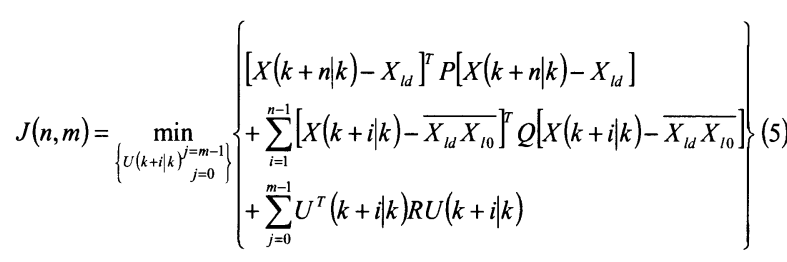

The first term of (5) refers to reaching the local desired coordinates, $X_{l d}=\left(x_{d} y_{d}, \theta_{d}\right)$. The second one is related to the distance between the predicted robot positions and the trajectory segment $\overline{X_{l d} X_{l 0}}$ given by a straight line between the initial robot coordinates $X_{l 0}=\left(x_{l 0}, y_{l 0}, \theta_{l 0}\right)$ from where the local perception frame was acquired, and the desired local position $X_{l d}=\left(x_{l d}, y_{l d}, \theta_{l d}\right)$ belonging to the local perception. The last one is related to the input signals denoted as $U$. The parameters $P, Q$ and $R$ are weighting parameters that express the importance of each term.

\section{B. LMPC algorithms ans simulation results}

By using the ideas previously introduced, it is presented here the LMPC algorithms that can be run in the following steps:

- Read the actual position.

- Minimize the cost function and obtain a series of optimal input signals.

- Choose the first obtained optimal input signal as the command signal.

- Go back to the first step in the next sampling period.

Minimizing the cost function is a convex optimization problem [20]; it is caused by the trigonometric functions used in the odometer system. The use of interior point methods can solve the above problem. Among many algorithms of optimization, the gradient descent algorithm was implemented in this work. To obtain the optimal solution, some constraints on the inputs are considered:

- The signal increment is kept fixed within the control horizon.

- The input signals remain constant during the remaining interval of time.

The above considerations can reduce the computation time and result in the smooth behaviour of along the prediction horizon [21]. Thus, the set of available inputs is reduced to one value. In order to reduce the search for an optimal signal value that minimizes the LMPC cost function, the possible input sets are considered as a bi-dimensional array. The array is decomposed into four zones. The search consists in analyzing the central points of each zone. It is considered only the region that offers the better optimization, where the algorithm is repeated for each sub-zone, until no sub-interval can be found. Once the algorithm was proposed, several simulations were carried out to verify its effectiveness, and then to make improvement. Thus, when only the desired coordinates are considered and the motors have different gain, the inputs can minimize the cost function by shifting the robot position. This problem can be easily solved by considering a straight-line trajectory from the current point of the robot to the final desired point. Thus, the trajectory should be included 5) in the LMPC cost function. The prediction horizons between $0.5 \mathrm{~s}$ and $1 \mathrm{~s}$ were proposed and the computation time for each LMPC step was set to less than $100 \mathrm{~ms}$, running in an embedded PC of $700 \mathrm{MHz}$. The computing time for the complete search of an optimal input is between 13 and $14 \mathrm{~ms}$ when $m=3$ and $n=5$. Where $m$ is the control horizon, and $n$ is the prediction horizon. In the case of using gradient descent method, the computing time is set to less than $1 \mathrm{~ms}$.

\section{LMPC experimental results}

The trajectory tracking performance is improved by the adequate choice of a cost function that is derived from (5) and consists of a quadratic function containing some of the following four parameters to be minimized: 
- The squared Euclidean approaching distance between the local desired coordinates, provided by the on-robot perception system, and the actual robot position.

- The squared trajectory deviation distance between the actual robot coordinate and a straight line that goes from the robot coordinates, when the local frame perception was acquired, and the local desired coordinates belonging to the referred frame of perception.

- The third parameter refers to changes allowed to the input signal.

- The last parameter consists in the squared orientation difference that is used only when the desired orientation is greater than a selected threshold. In this case, other parameters of the cost function are not used.

One consideration that should be taken into account is the different distance magnitudes. In general, the approaching distance could be more than one meter. However, the deviation distance has its magnitude normally in the order of $\mathrm{cm}$, which becomes effective only when the robot is approaching to the final desired point. Hence, when it is tried to reduce further the deviation distance to less than $1 \mathrm{~cm}$, it is proposed to increase the weight value for the deviation distance in the cost function. The trajectory tracking accuracy is an important aspect to be considered. In this context, the odometer system performance was analyzed by measuring the accuracy of the system. It was done by commanding long trajectories along lab corridors. After calibrating the odometer, the results showed that a commanded trajectory of $22 \mathrm{~m}$ provided averaged final distance errors of less than $0.5 \mathrm{~m}$, and angular orientation errors of less than $5^{\circ}$. Hence in this research, it is analyzed local trajectories of less than $1.5 \mathrm{~m}$ accordingly with the narrow visual perception provided. Thus, the odometer system errors can be neglected when local trajectories are considered. Therefore, the odometer system is locally used to compute LMPC trajectory tracking errors. The tested trajectories are obtained from the available set of local map coordinates as shown in Figure 2.

The LMPC results are analyzed when different trajectories tracking are commanded, as it is depicted in Figure 4. Denote $E_{1}$ as the average final error, $E_{2}$ the maximal average tracking error, $E_{3}$ the average tracking error and $E_{4}$ the standard deviation of average tracking error. Table 2 presents the statistics concerning about the error obtained in $\mathrm{cm}$ testing the trajectories shown in Figure 4. It can be seen that the accuracy of trajectory tracking, when straight line is commanded, has a deviation error of $0.54 \mathrm{~cm}$. However, when a turning action is performed, the error in straight line tracking is bigger as consequence of the robot dynamics when it is moving forward. Hence, the forward movement consists in usually a steering action. Figure 4 gives a clue about what is happening. Thus, the major turning angle will produce the major deviation distance. Usually, it is very difficult to reduce the approaching distance to zero, due to the control difficulty of dead zone for the WMR and to the fact that the final target is considered in the present work as being reached by the robot when the Euclidean approaching distance is less than $5 \mathrm{~cm}$.

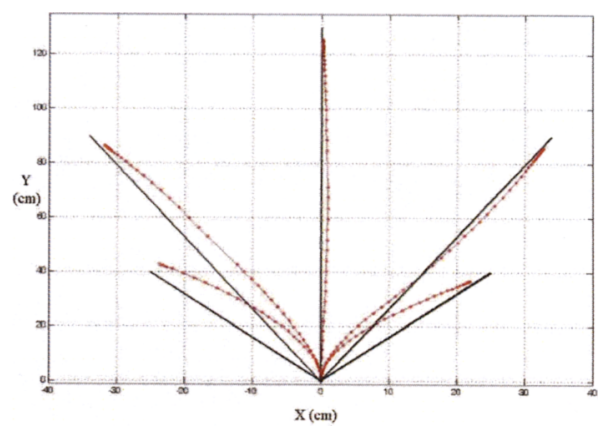

Fig.4. Different trajectories

Table 2. Point to point trajectory tracking statistics

\begin{tabular}{lcccc}
\hline \multicolumn{1}{c}{ Trajectory } & $\boldsymbol{E}_{\boldsymbol{I}}$ & $\boldsymbol{E}_{2}$ & $\boldsymbol{E}_{3}$ & $\boldsymbol{E}_{4}$ \\
\hline $\begin{array}{l}\text { From }(0,0) \text { to } \\
(0,130)\end{array}$ & $4.4 \mathrm{~cm}$ & $0.9 \mathrm{~cm}$ & $0.54 \mathrm{~cm}$ & $0.068 \mathrm{~cm}$ \\
\hline $\begin{array}{l}\text { From }(0,0) \text { to } \\
(34,90)\end{array}$ & $3.8 \mathrm{~cm}$ & $3.9 \mathrm{~cm}$ & $2.3 \mathrm{~cm}$ & $0.82 \mathrm{~cm}$ \\
\hline $\begin{array}{l}\text { From }(0,0) \text { to } \\
(25,40)\end{array}$ & $4.5 \mathrm{~cm}$ & $5.3 \mathrm{~cm}$ & $3.9 \mathrm{~cm}$ & $1.96 \mathrm{~cm}$ \\
\hline
\end{tabular}

Other interesting results consist in testing the LMPC performance when the trajectory is composed of a set of points to be tracked. Due to the narrow perception system used only continuous movement in advancing sense is implemented. The trajectory tracking strategy with continuous movement, for a set of points $(0,0),(25,50),(25,100),(0,150)$ and $(0,200)$, is represented in Figure 5. In this case, the reported larger trajectory deviation is due to the WMR's mechanical dynamics.

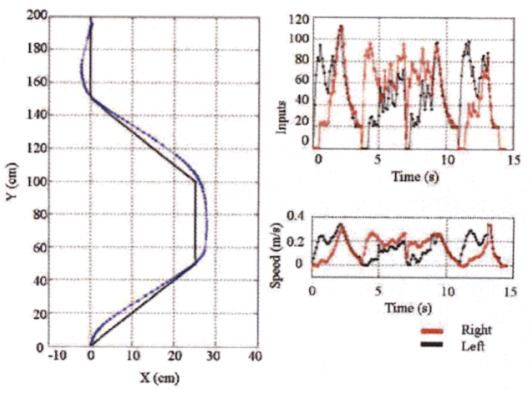

Fig. 5. A specific trajectory

\section{NAVIGATION RESULTS}

In this section is tested the previously introduced navigation strategy. It should be noted that due to some false positive detection problems, reported in [22], the robot is positioned from local desired point to local desired point and then the frame is acquired. Therefore, frames containing false positives can be discarded and the path planning strategy can be successfully tested. Figure 6 reports an experiment, which consists in changing the WMR configuration from $(0,0)$ to $(0$, $650 \mathrm{~cm}$ ) in a $3 \mathrm{~m}$ wide corridor in which multiple obstacles have been placed to test the reactive behavior and flexibility. The experimental results show that local reactive navigation can accomplish with a more global desired goal by using the presented strategy. 


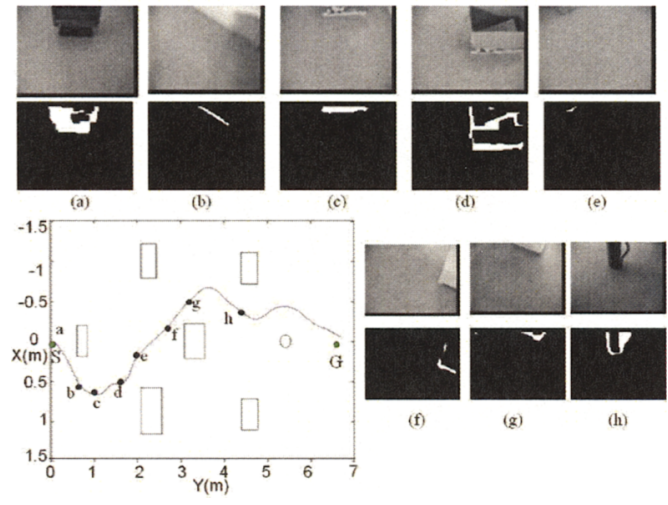

Fig. 6. Trajectory in a $3 m$ wide corridor

Therefore, LMPC and local monocular perception are used to control and to obtain approaching trajectories towards the desired configuration by the mean of artificial attraction potential fields. Finally, the problem of wheel slippage should be mentioned. However, in this work the final error reported, by using the Euclidean distance, was less than $25 \mathrm{~cm}$ in the experiment shown. In this sense, the methodology presented allows autonomous WMR navigation with a certain degree of freedom, where the use of landmarks can minimize dead reckoning problems. However, considering the results obtained a complete environment map can be avoided. Of course, we did more experiments in indoor environments that we don't place in the paper for lack of available space.

\section{CONCLUSIONS}

The research reported have provided remarkable successes even considering the perception limitations explored in this work. Potential field attraction points have shown their effectiveness dealing with reactive behaviours in structured indoor environments. Trajectory tracking performance using LMPC techniques has provided good results, with final position local errors less than $5 \mathrm{~cm}$. Moreover, contractive constraints have assured a convergence criterion towards the different commanded points. Hence, satisfactory results can be obtained with trajectories of nearly $20 \mathrm{~m}$ or even longer. Therefore, a new navigation paradigm has been explored in which local navigation in partially unknown environments can be performed with a certain degree of freedom, when effective attraction potential fields are commanded.

The future work is mainly addressed to improve the present sensorial system. Therefore research will be focused on the use of a tessellated probabilistic representation within a local occupancy grid framework by using the floor model and time integration image sequences. Thus, it is expected to remove some false obstacles detected. Moreover, the use of obstacle models can be considered as a clue in order to find natural landmarks that can provide a way to minimize dead reckoning drawbacks and provide feasible WMR localizations along larger scenarios where a map description is necessary. Local minimal failures caused usually by dynamic moving objects (as human movement) should be also analyzed, especially when effective potential fields are selected. Hence, in some cases stopping the robot is not a bad strategy as i.e. when the interaction human-WMR is pursued.

\section{ACKNOWLEDGMENT}

This work has been partially funded by the Commission of Science and Technology of Spain (CICYT) through the coordinated project DPI-2007-66796-C03-02, and by the Government of Catalonia through the Network Xartap and the consolidated research group's grant SGR2005-01008.

\section{REFERENCES}

[1] Last, D. (2008) Who are the Navigators?, The Journal of Navigation, 61, $1-6$.

[2] Moravec, H. P. \& Elfes, A. (1985) High Resolution Maps from Wide Angle Sonar. Proc. IEEE Int'l Conf. Robotics and Automation, 116-121.

[3] Borenstein, J. \& Koren, Y. (1989) Real-Time Obstacle Avoidance for Fast Mobile Robots. IEEE Trans. System, Man, and Cybernetics, 19 (5), 1179 1187.

[4] DeSouza, G. N. \& Kak, A. C. (2002) Vision for Mobile Robot Navigation: A Survey. IEEE Transactions on Pattern Analysis and Machine Intelligence, 24 (2), 237 - 267.

[5] Thrun, S. (2000) Probabilistic Algorithms in Robotics. Technical Report CMU-CS-00-126, Carnegie Mellon University.

[6] Kosaka, A. \& Kak, A. C. (1992) Fast Vision-Guided Mobile Robot Navigation Using Prediction of Uncertainties. Computer Vision, Graphics, and Image Processing-Image Understanding, 56 (3), 271-329.

[7] Kuipers, B. J., Byun, Y. T. (1991) A Robot Exploration and Mapping Strategy Based on a Semantic Hierachy of Spatial Representation. J. Robotics and Autonomous Systems, 8, 47-63.

[8] Hashima, M., Hasegawa, F., Kanda, S., Maruyama, T., Uchiyama, T. (1997) Localization and Obstacle Detection for a Robot Carrying Food Trays. Proc. IEEE Int'l Conf. Intel. Robots and Systems, 345-351.

[9] Butler, Z. (2006) Corridor planning for natural agents, Proc. IEEE International Conference on Robotics and Automation, 499-504.

[10] Pacheco, L., Luo, N., Ferrer, I., Cufi, X. (2008) Control Education within a Multidisciplinary Summer Course on Applied Mobile Robotics, Inter. Federation of Automatic Control World Congress, (in press).

[11] Horn, B. K. P. (1998). Robot Vision. McGraw -Hill Book Co., Twelfth printing, New Jersey.

[12] Campbell J., Sukthankar R., Nourbakhsh I. (2004) Techniques for Evaluating Optical Flow in Extreme Terrain. Proc. of IEEE Int. Conf. on Intel. Robots and Systems, 4, 3704- 3711.

[13] Nourbakhsh I. R., Andre D., Tomasi C., Genesereth M. R. (1997) Mobile Robot Obstacle Avoidance Via Depth From Focus, Robotics and Autonomous Systems, 22, pp. 151-58.

[14] Fox, D., Burgard, W., and Thrun, S. (1997) The dynamic window approach to collision avoidance, IEEE Robotics and Automation Magazine, 4, 23-33.

[15] Ögren, P., Leonard, N. (2005) A convergent dynamic window approach to obstacle avoidance, IEEE Transaction on Robotics, 21(2), 188-195.

[16] Thrun, S. (2002) Robotic mapping: a survey, Exploring Artificial Intelligence in the New Millennium, Morgan Kaufmann, San Mateo, CA.

[17] Gupta, G.S., Messom, C. H., Demidenko, S., (2005) Real-time identification and predictive control of fast mobile robots using global vision sensor, IEEE Trans. on Instr. and Measurement, 54(1), 200-214.

[18] Küne, F., Lages, W., Silva, J., (2005) Point stabilization of mobile robots with nonlinear model predictive control, Proc. IEEE Int. Conf. On Mech. and Aut., 1163-1168.

[19] Pacheco, L. \& Luo, N. (2007) Mobile Robot Local Predictive Control Using a Visual Perception Horizon, Int. Journal of Factory Autom. Robotics and Soft Comp., I(2), 73-81.

[20] Boyd, S. \& Vandenberghe, L. (2004) Convex Optimization, Cambridge University Press.

[21] Maciejowski, J. M. (2002) Predictive Control with Constraints, Ed. Prentice Hall Int.

[22] Pacheco, L., Cufi, X., Cobos, J. (2007) Constrained Monocular Obstacle Perception with Just One Frame, Lecture Notes in Computer Science, Springer-Verlag, Pattern Recognition and Image Analysis, J. Marti et al. (Eds), 1, 611-619. 